Classification

Physics Abstracts

42.60F

\title{
Utilisation de lasers multi-electrodes en tant que mélangeur hyperfréquence
}

\author{
R. Hamelin $\left({ }^{1}\right)$, J.P. Vilcot $\left({ }^{1}\right)$, J.P. Gouy $\left({ }^{1}\right)$, D. Decoster $\left({ }^{1}\right)$, C. Carriere $\left({ }^{2}\right)$, B. Groussin $\left({ }^{2}\right)$ \\ et V. Cargemel $\left({ }^{2}\right)$ \\ ( ${ }^{1}$ Institut d'Electronique et de Microélectronique du Nord, UMR-CNRS 9929-Département \\ Hyperfréquence et Semiconducteurs, Domaine Universitaire et Scientifique de Villeneuve d'Ascq, \\ Avenue Poincaré, BP. 69, 59652 Villeneuve d'Ascq Cedex, France \\ $\left({ }^{2}\right)$ Thomson CSF semiconducteurs spécifiques-Dept. Optoélectronique, Route départementale \\ 128, BP. 48, 91401 Orsay Cedex, France
}

(Reçu le 4 juillet 1994, révisé le 15 novembre 1994, accepté le 21 décembre 1994)

Résumé. - Le but de cet article est de décrire une approche technologique améliorée, pour la fabrication de lasers multi-électrodes de type Fabry-Pérot pour des applications hyperfréquences. Seuls deux masques sont nécessaires, et nous utilisons exclusivement la photolithographie optique. Nous préciserons les motivations des différents choix technologiques et nous présenterons les premiers résultats d'une utilisation originale de ces lasers en tant que mélangeur hyperfréquence.

\begin{abstract}
The purpose of this paper is to describe a new technological process for the fabrication of Fabry-Pérot multi-electrodes lasers. Only two masks are required and we use selfalignement technics and exclusively optical photolithography. We will precise the motivation of the different technological choices that have been done and we will present the first results of an original use of these components as a microwave mixer.
\end{abstract}

\section{Introduction}

Les lasers multi-électrodes ont été largement étudiés pour leur intérêt dans la génération d'impulsions courtes [1], l'auto-pulsation [2] ou encore pour leurs propriétés intéressantes dans le domaine des hyperfréquences [3]. Nous proposons donc une manière simple et rapide de fabriquer ces composants, en nous intéressant à quelques propriétés hyperfréquences. La façon la plus simple de fabriquer un laser de type Fabry-Pérot est d'opter pour une structure de type "ridge", car aucune reprise d'épitaxie n'est nécessaire, de plus cette structure a déjà prouvé ses propriétés [4]. Nous proposons une structure planarisée avec une technologie où seulement deux étapes de photolithographie sont mises en jeu sans alignement critique.

En ce qui concerne les caractéristiques de modulation il est évident que l'on doit minimiser les capacités des plots de contacts. C'est pourquoi, afin d'éviter une coupure fréquentielle de 
type circuit, nous avons déposé des plots de contact de faible surface sur une forte épaisseur de polyimide (matériau de faible constante diélectrique). Ce polymère nous permet de surcroît de planariser la structure de type "ridge". Cependant, un problème classique dans l'utilisation des polyimides est leur ductilité lors du clivage. Il est donc nécessaire de retirer le polymère sur une surface maximale. Ce revêtement de surface ne pouvant pas jouer le rôle de passivant pour l'AlGaAs exposé après gravure, nous passiverons le composant avant le dépôt du polymère.

\section{Principe de base}

Le schéma de principe du laser multi-électrode étudié est représenté figure 1 . Il est constitué de deux électrodes de longueurs différentes dont la plus grande est a priori utilisée pour pomper le laser (électrode de pompe) ; la plus courte isolée électriquement de l'autre serait plutôt utilisée pour appliquer le signal hyperfréquence de modulation de l'onde optique (électrode de commande). Il a récemment été montré qu'une telle façon de procéder pourrait conduire à une amélioration significative de la fréquence de coupure des lasers hyperfréquence [3]. Nous avons donc envisagé la réalisation technologique d'un tel dispositif afin d'en vérifier l'intérêt pour les hyperfréquences.

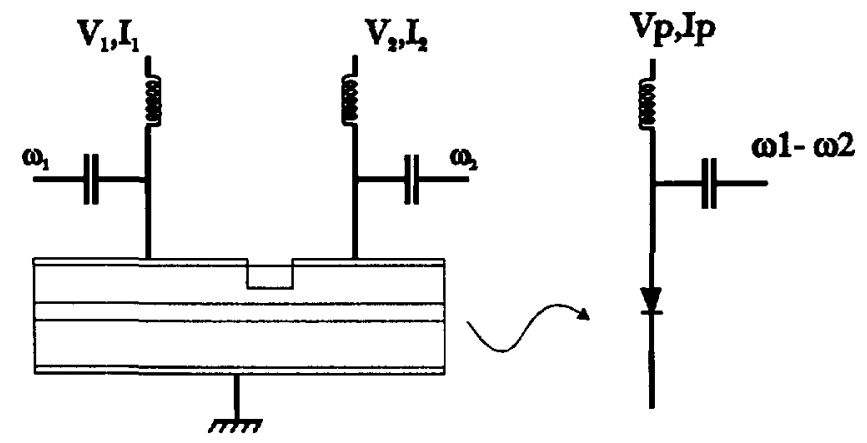

Fig. 1. - Schéma de principe du composant étudié.

[Schematic view of the studied device.]

De par l'existence d'une résistance parasite entre les deux électrodes, le fait d'appliquer une tension sur l'une ou l'autre des électrodes entraîne une polarisation de la seconde. Dans notre cas, il nous est alors impossible de polariser l'électrode de commande à des valeurs comprises entre 0 et $0,9 \mathrm{~V}$. Nous verrons également que l'application d'une tension inverse sur cette même électrode entraîne la génération d'un signal dont la fréquence est de l'ordre du GHz, par conséquent toutes les analyses dynamiques ont été effectuées à des tensions comprises entre 0,9 et $1,7 \mathrm{~V}$.

En outre, le fait de disposer de deux accès hyperfréquence nous incite à penser que ce composant pourrait être un dispositif de choix pour effectuer le mélange de deux signaux hyperfréquences différents. En effet, il est naturel de penser que la caractéristique $P(I)$ d'un tel laser (où le courant $I$ est celui injecté dans l'électrode de pompe) sera fonction de la valeur de la tension appliquée sur l'électrode de commande. On peut ainsi imaginer que le signal optique issu du laser sera proportionnel au produit des signaux de fréquences $f_{1}$ et $f_{2}$ appliqués sur chaque électrode. Dans ces conditions, on devrait récolter non seulement des signaux aux 
fréquences $f_{1}$ et $f_{2}$ mais également aux fréquences $\left(f_{1}-f_{2}\right)$ et $\left(f_{1}+f_{2}\right)$, concrétisant ainsi le concept de mélange. On devrait s'attendre dans ces conditions à ce que l'intensité des signaux à la fréquence $\left(f_{1}-f_{2}\right)$ et $\left(f_{1}+f_{2}\right)$ soient du même ordre de grandeur que les signaux de fréquence $f_{1}$ et $f_{2}$. Nous avons vérifié cette possibilité.

\section{Technologie}

La structure épitaxiale utilisée dans cette étude est décrite en figure 2. Il s'agit d'une structure laser à zone de confinement séparée à un puits quantique d' $\mathrm{Al}_{0,1} \mathrm{Ga}_{0,9} \mathrm{As}$, réalisés dans les laboratoires de Thomson TCS par Epitaxie en Phase Vapeur aux Organo-Métalliques (EPVOM) basse pression.

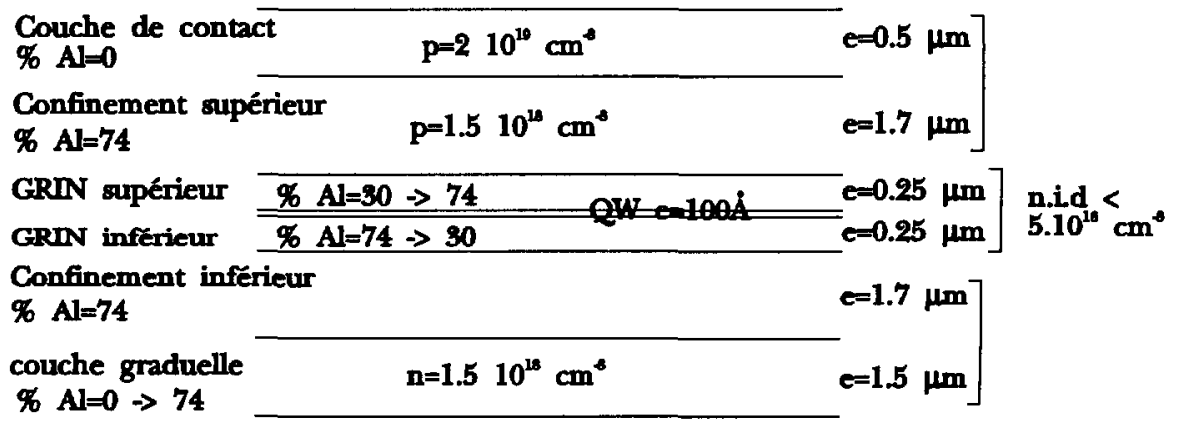

\section{Substrat GaAs de type $n$}

Fig. 2. - Structure épitaxiale.

[Epitaxial structure.]

La première étape de fabrication est la définition du guide d'onde par gravure chimique d'un ruban de $6 \mu \mathrm{m}$ de large le long de la direction [110] par photolithographie standard (cf. Fig. 3). La solution utilisée est $\mathrm{H}_{2} \mathrm{SO}_{4}: \mathrm{H}_{2} \mathrm{O}_{2}: \mathrm{H}_{2} \mathrm{O}(1: 1: 18)$. L'attaque est arrêtée $0,2 \mu \mathrm{m}$ avant la zone de confinement. Après avoir décapé, on dépose une couche de $2000 \AA$ de nitrure par PECVD sur toute la surface afin de passiver l'AlGaAs exposé. Par la suite, du polyimide est déposé à la tournette et recuit sous atmosphère neutre. Grâce à la viscosité des polyimides, la couche déposée est plus épaisse à la base du mésa qu'au sommet; nous passons d'un guide d'onde de $1,8 \mu \mathrm{m}$ de hauteur à un dénivelé de $0,9 \mu \mathrm{m}$. Nous procédons ensuite à la planarisation de l'édifice en superposant une forte épaisseur de résine (environ $3 \mu \mathrm{m}$ ). Le dénivelé n'est alors plus que de $0,2 \mu \mathrm{m}$. Une gravure sèche par plasma $\mathrm{O}_{2}$ de la totalité de la structure va planariser le composant. Les vitesses de gravure du polyimide et de la résine sont comparables, ce qui nous permet de conserver le même dénivelé tout au long de la gravure. Un échantillon témoin sert de référence pour connaître les différentes épaisseurs de polymères déposés au sommet et à la base du guide d'onde ce qui fixe précisément le temps de gravure. On arrête l'attaque sur le nitrure qui recouvre la surface de contact, une attaque plasma $\mathrm{CF}_{4}$ est alors nécessaire pour exposer la couche de contact de GaAs. La structure est planarisée et le second masquage à lieu, les contacts ohmiques de type $\mathrm{p}$ sont alors déposés par évaporation de la séquence $\mathrm{Pt} / \mathrm{Ti} / \mathrm{Pt} / \mathrm{Au}(100 / 400 / 100 / 3000 \AA)$. Après "lift-off", la métallisation est utilisée en tant que masque afin de retirer le polyimide de la quasi totalité de la surface. Cette gravure au plasma 

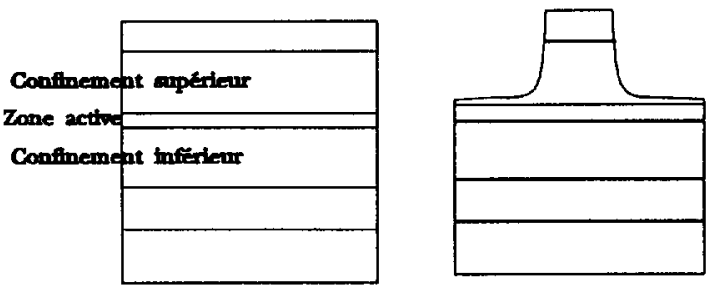

Définition du guide

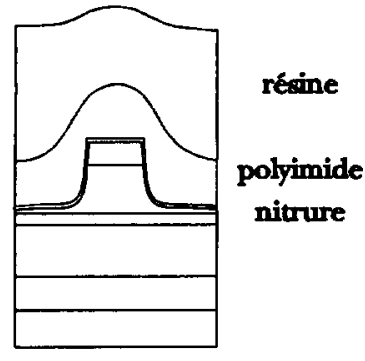

Passivation

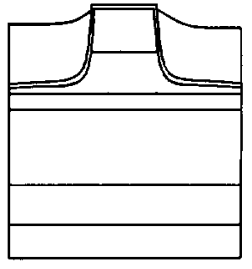

Planarisation (plasma $O_{2}$ )

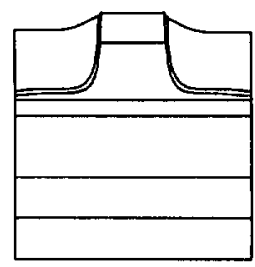

Ouverture du nitrure par attaque plasma CF,

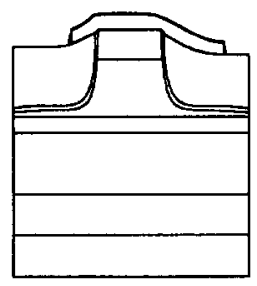

Dépôt des contacts ohmiques de type $p$

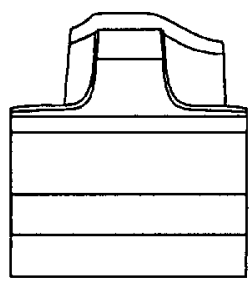

Attaque plasma $O$, auto-allignée

Fig. 3. - Processus technologique.

[Technological process-flow.]

$\mathrm{O}_{2}$ n'est pas critique car l'attaque s'arrête naturellement sur la couche de nitrure. Il ne reste du polyimide que sous les métallisations ce qui ne pose aucun problème lors du clivage. L'isolation électrique des électrodes s'obtient par la gravure chimique de la couche de contact, cette dernière est auto-alignée étant donnée que la seule surface encore exposée est l'espace inter-électrode, cette étape ne nécessite pas de masquage supplémentaire. Une résistance parasite de $1,3 \mathrm{k} \Omega$ est mesurée typiquement pour un espace inter-électrode de $6 \mu \mathrm{m}$., Une gravure plus profonde dans l'AlGaAs n'augmente pas cette résistance. Suivent alors les opérations d'amincissement et de métallisation des contacts ohmiques de type $\mathrm{n}$ sur la face arrière par l'évaporation de la séquence $\mathrm{Ni} / \mathrm{Ge} / \mathrm{Au} / \mathrm{Ti} / \mathrm{Au}(150 / 195 / 390 / 500 / 2000 \AA)$. Les contacts sont alliés par un même recuit rapide à $400{ }^{\circ} \mathrm{C}$ pendant $40 \mathrm{~s}$. La position du clivage déterminera les dimensions et la localisation des électrodes. L'électrode de pompe mesure entre 200 et $400 \mu \mathrm{m}$ de long, l'électrode de commande mesure entre 20 et $40 \mu \mathrm{m}$, l'espace inter-électrode est de $6 \mu \mathrm{m}$.

\section{Comportement sous polarisation continue}

Dans des conditions de polarisation homogène et continue nous mesurons un seuil de $20 \mathrm{~mA}$ pour des cavités de $300 \mu \mathrm{m}$, et une efficacité externe de l'ordre de $0,6 \mathrm{~W} / \mathrm{A}$. Lorsque l'électrode de commande ("petite" élèctrode : $20 \mu \mathrm{m}$ ) n'est raccordée à aucun circuit extérieur nous pouvons noter un décrochement sur la courbe $P(I)$ (cf. Fig. 4) ; elle joue ici le rôle d'absorbeur saturable et sa petite taille entraine une saturation rapide et l'on ne peut donc noter aucune hystérésis. Lorsque cette seconde électrode est raccordée à un circuit extérieur, les porteurs photocréés dans cette zone de polarisation faible peuvent être évacués ; l'absorbeur ne sature 


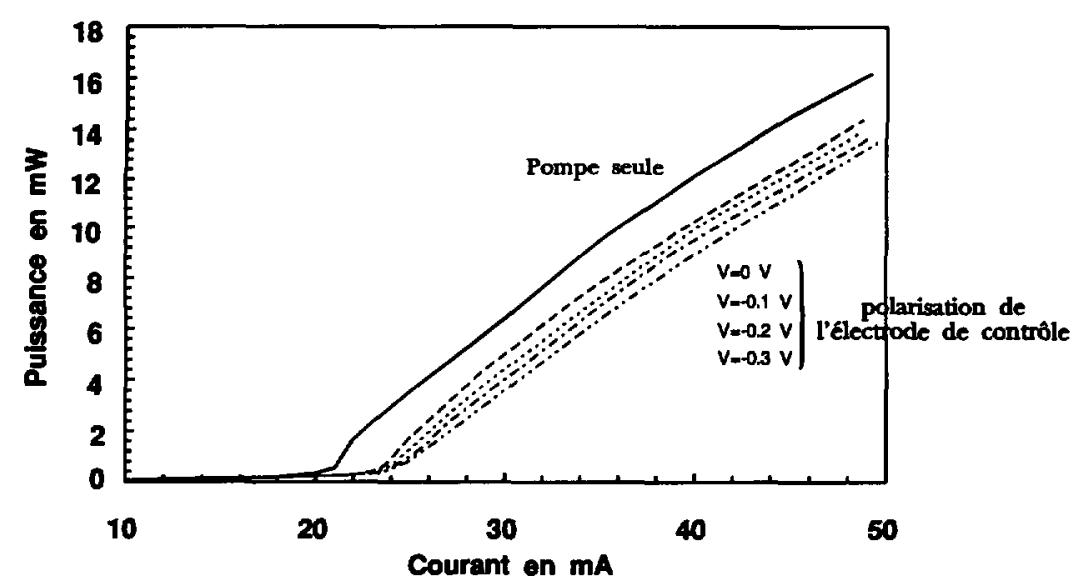

Fig. 4. - Comparaison des caractéristiques statiques pour différentes tensions de controle. [Comparison of the static properties under different biasing voltage of the short electrode.]

plus et le décrochement n'est alors plus visible sur les caractéristiques $P(I)$. Dans certains cas de polarisation, et notamment sous polarisation négative, ces phénomènes de génération de porteurs et d'évacuation peuvent donner lieu à la génération spontanée d'un signal de l'ordre du $\mathrm{GHz}$ sous des conditions d'injection continue, la fréquence de ces oscillations dépendant de la tension appliquée et de la durée de vie des porteurs. Ces auto-oscillations ne représentent malheureusement pas une source hyperfréquence "propre" comme nous le montre la figure 5a, cependant il est possible, en appliquant une très faible modulation sur l'électrode de commande, d'affiner la largeur spectral du signal (Fig. 5b). Ce comportement instable en polarisation négative de l'électrode de commande interdit tout contrôle dynamique du composant de par la présence de ces oscillations parasites. Lorsque l'électrode de commande est polarisée en direct, la résistance inter-électrode agit comme un diviseur de tension et la zone absorbante est "autopolarisée". Dans ces conditions, les porteurs photogénérés ne sont plus collectés par le circuit extérieur et les deux parties du laser peuvent être modulées de façon indépendante.

\section{Propriétés hyperfréquences}

Lorsque la polarisation dépasse le seuil électrique de la diode constituée par l'électrode de contrôle, un courant est injecté, l'auto-oscillation disparaît et l'on peut alors moduler le laser par l'une ou l'autre des électrodes (cf. Fig. 6). Sur l'électrode de commande, il s'agit d'une modulation du gain et non de l'absorption saturable, c'est pourquoi aucune amélioration de l'efficacité de la modulation ne peut être observée, on peut néanmoins constater une légère amélioration de la caractéristique dynamique du composant, qui pourrait être attribuée à la réduction de la durée de vie des photons dans cette partie du laser qui est plus courte. Notons par ailleurs, que les conditions de polarisations de l'électrode de commande sont telles que la résistance différentielle dynamique de la diode est proche de $50 \Omega$; le dispositif se trouve donc naturellement adapté et le transfert de la puissance hyperfréquence devrait être plus efficace. La capacité est typiquement de $4 \mathrm{pF}$ pour l'électrode de pompe et de $0,4 \mathrm{pF}$ pour l'électrode de commande. La capacité des plots de contacts est ici négligeable.

Le concept de mélangeur micro-onde à l'aide de laser multi-électrodes a été mis en oeuvre sur un laser de cavité de $230 \mu \mathrm{m}$, comportant deux électrodes et de distance inter-électrode de $6 \mu \mathrm{m}$. 


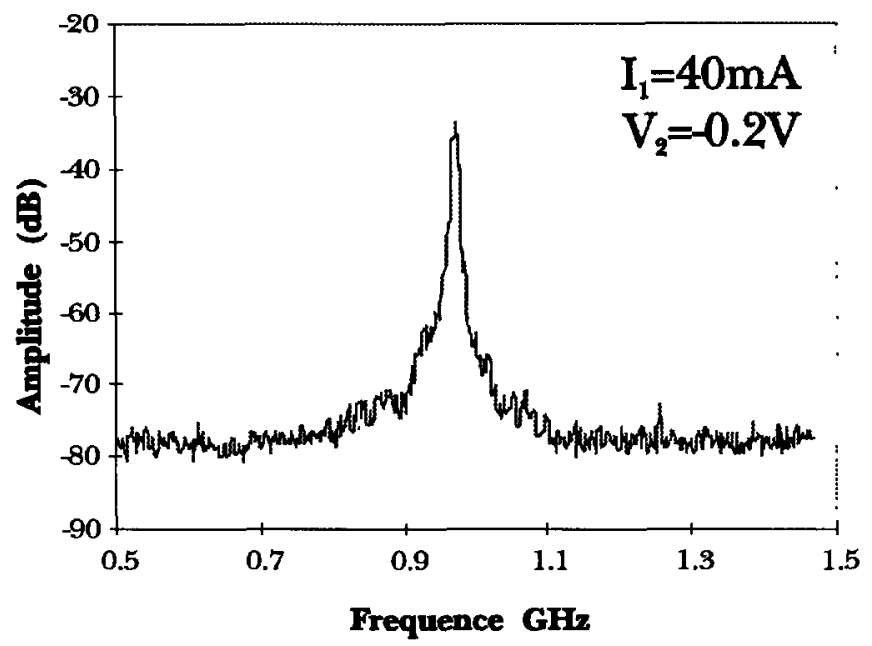

a)

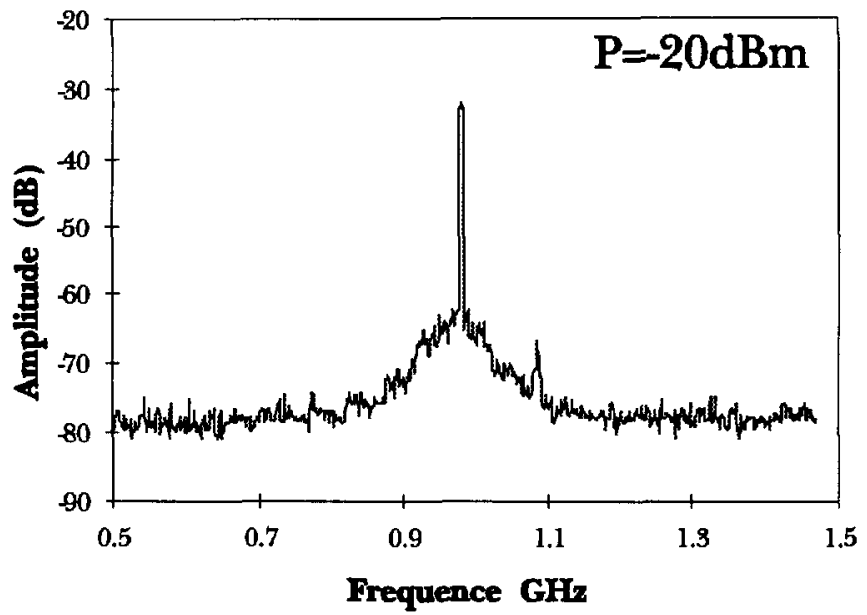

b)

Fig. 5. - a) Auto-oscillation à $1 \mathrm{GHz}$. b) Contrôle de la largeur spectrale du signal auto-oscillant par un signal externe.

[a) Self-oscillation at $1 \mathrm{GHz}$. b) Control of the spectral width of the self-oscillating signal by an external signal.]

L'électrode de l'absorbeur saturable est de $20 \mu \mathrm{m}$ de long. Nous avons exploré les fréquences comprises entre 1 et $2,5 \mathrm{GHz}$ à l'aide de deux signaux de fréquences $f_{1}$ et $f_{2}$ séparés de $100 \mathrm{MHz}$ appliqués séparément sur les électrodes pour des puissances micro-ondes de $-5 \mathrm{dBm}$. Nous avons mesuré sur analyseur de spectre les signaux issus d'une photodiode PIN rapide. Les résultats expérimentaux montrent l'existence d'un signal à la fréquence $\left(f_{1}-f_{2}\right)$ dont le niveau est comparable aux signaux de fréquence $f_{1}$ et $f_{2}$, ainsi que le montre la figure 7 qui donne le rendement de conversion d'un tel système. Notons que l'allure de cette courbe rappelle la réponse dynamique de notre composant. Le signal à la fréquence $f_{1}+f_{2}$ n'apparaît pas compte tenu de la fréquence de coupure du laser, la figure 8 montre néanmoins l'existence de ce signal dans le cas de faibles fréquences. Alors que dans un mélangeur conventionnel on utilisera les non linéarités d'un composant pour générer des harmoniques à partir de la somme de deux signaux, on voit que dans notre cas, la simple modulation mutuelle des deux signaux appliqués aboutit 


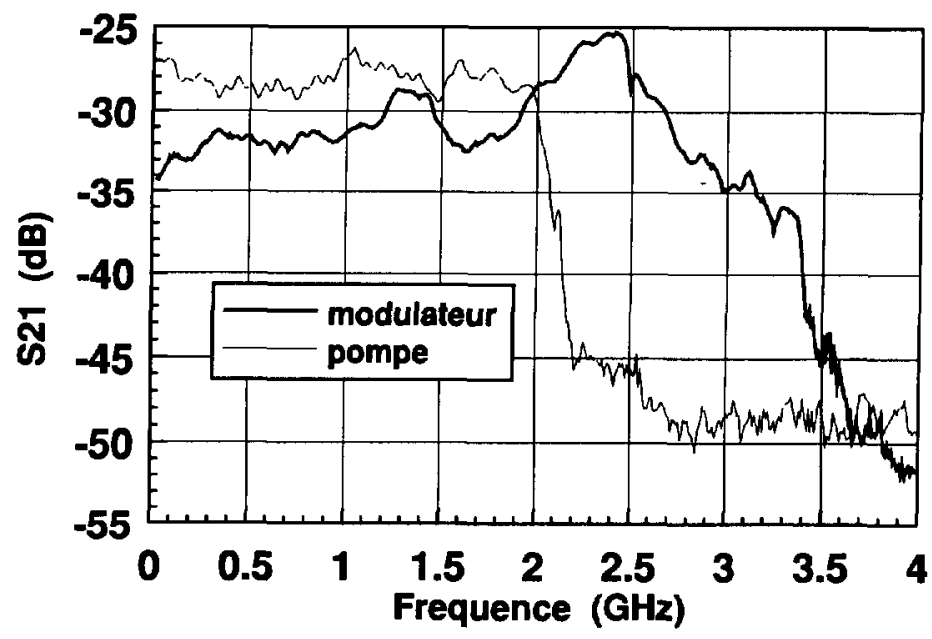

Fig. 6. - Comparaison des propriétés dynamiques de chacune des électrodes courant de pompe $35 \mathrm{~mA}$, tension de contrôle $1,7 \mathrm{~V}$.

[Comparison of the dynamic properties of each electrode, pumping current $35 \mathrm{~mA}$, short electrode biasing voltage $1.7 \mathrm{~V}$.]

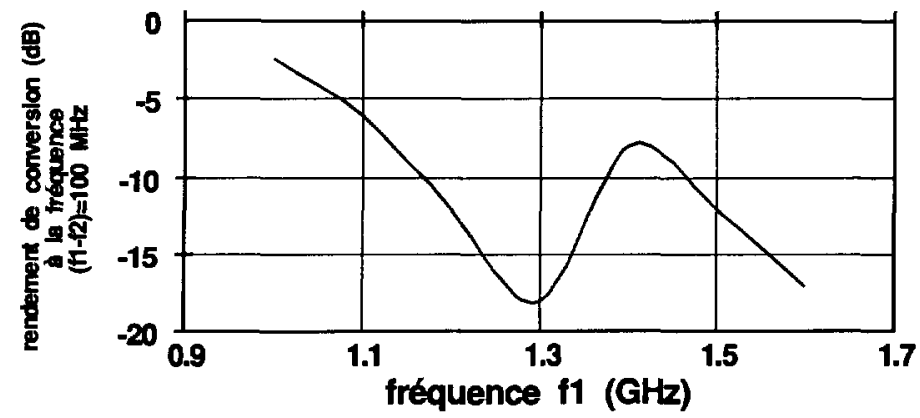

Fig. 7. - Rendement de conversion en fonction de la fréquence du signal $f_{1}$ pour un écart $\left(f_{1}-f_{2}\right)$ de $100 \mathrm{MHz}$. Courant de pompe : $40 \mathrm{~mA}$. Petite électrode autopolarisée.

[Conversion efficiency as a function of the signal of frequency $f_{1}$ for $\left(f_{1}-f_{2}\right)=100 \mathrm{MHz}$. Pumping current $40 \mathrm{~mA}$. Short electrode self-biased.]

au même résultat. Une amélioration du rendement de conversion peut alors être envisagée en optimisant la géométrie du composant (longueur de cavité, rapport de longueurs des électrodes, efficacité de modulation ou encore le nombre d'électrodes).

\section{Conclusion}

Nous avons montré dans cette étude une nouvelle technologie de fabrication de lasers GaAs multi-électrodes ne comportant que deux étapes de masquage et ne faisant appel qu'à la photolithographie optique. Cette première réalisation nous a permis de vérifier les aptitudes de ce composant pour des applications hyperfréquences. Tout d'abord en tant qu'oscillateur, nous avons montré comment améliorer la qualité spectrale du signal généré par un pilotage externe. 


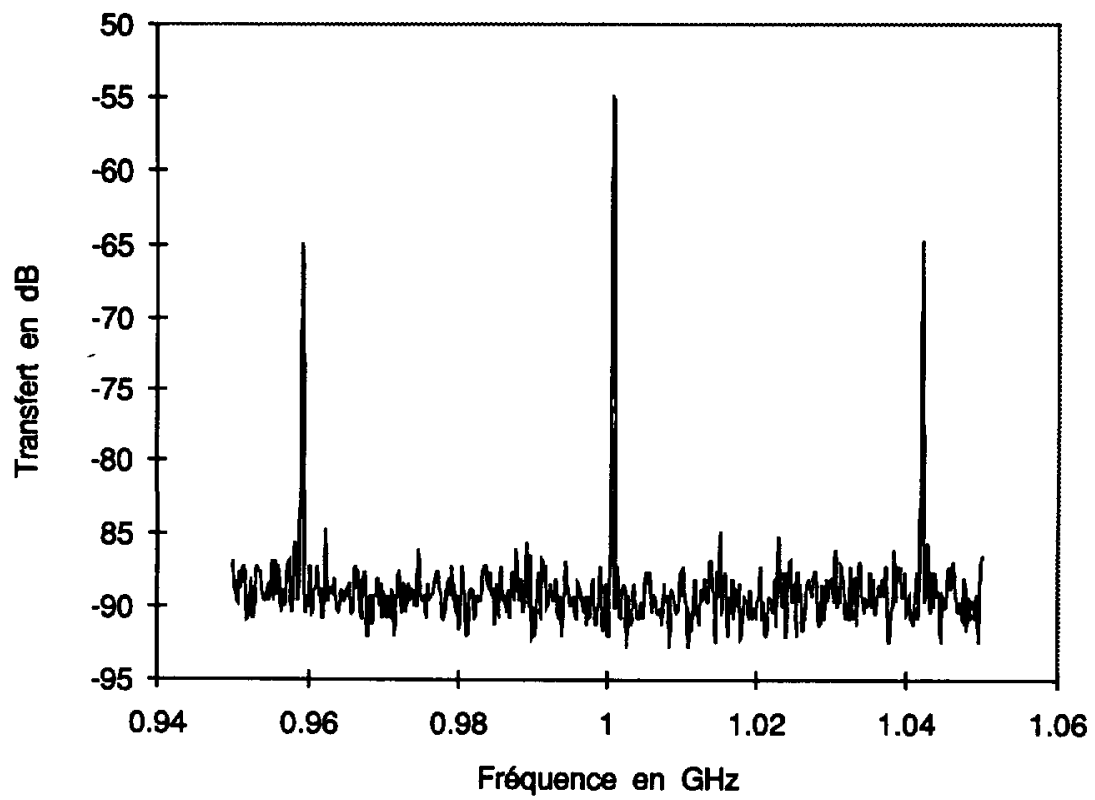

Fig. 8. - Spectre de mélange d'un signal à $1 \mathrm{GHz}$ appliqué sur l'électrode de commande (autopolarisée) et d'un signal à $40 \mathrm{MHz}$ appliqué sur l'électrode de pompe ( $I=35 \mathrm{~mA}$ ).

[Mixing of a signal at $1 \mathrm{GHz}$ applied on the command electrode (self-biased) and a signal at $40 \mathrm{MHz}$ applied on the long electrode $(I=35 \mathrm{~mA})]$

Ensuite par la séparation des fonctions de pompe et de modulation nous montrons une amélioration des caractéristiques dynamiques. Enfin nous présentons la possibilité d'utiliser les lasers multi-électrodes en tant que mélangeur hyperfréquence.

\section{Bibliographie}

[1] Chen Y.K., Wu M.C., Tanbun-Ek T., Logan R.A. et Chin M.A., Appl. Phys. Lett. 58 (1991) 1253-1255.

[2] Farrel G., Phelan P. et Hegarty J., Electron. Lett. 27 (1991) 1403-1404.

[3] Elkadi H., Vilcot T.P. et Decoster D., Microw. Opt. Technol. Lett. 6 (1993) 245-249 ; id., Microw. Opt. Technol. Lett. 7 (1994) en cours d'impression.

[4] Nagarajan R., Fukushima T., Bowers J.E., Geels S. et Coldren L.A., Appl. Phys. Lett. 58 (1991) 2326-28. 
Articles Réguliers

Regular Papers 